\title{
A Qualitative Investigation of the Barriers to Help-seeking Among Members of the Public Presented with Symptoms of New-onset Rheumatoid Arthritis
}

\author{
Gwenda Simons, Christian David Mallen, Kanta Kumar, Rebecca Jayne Stack, and Karim Raza
}

\begin{abstract}
Objective. Treating patients with rheumatoid arthritis (RA) within 3 months of symptom onset leads to significantly improved clinical outcomes. However, many people with RA symptoms wait a long time before seeking medical attention. To develop effective health interventions to encourage people to seek help early, it is important to understand what the general public knows about RA, how they would react to the symptoms of RA, and what might delay help-seeking.

Methods. Qualitative interviews were conducted with 38 members of the general public (32 women) without any form of inflammatory arthritis about their perceptions of RA symptoms and decisions to seek help were they to experience such symptoms. The interviews were audio-recorded, transcribed verbatim, and analyzed using thematic analysis.

Results. A number of barriers and drivers to help-seeking were identified and grouped into 5 themes: perceived causes of symptoms; factors related to presentation, location, and experience of symptoms; perceived effect of symptoms on daily life; self-management of symptoms; and general practitioner-related drivers and barriers.

Conclusion. To our knowledge, our study is the first to investigate barriers to and drivers of help-seeking in response to the onset of RA symptoms in individuals without a diagnosis of RA. It has revealed a number of additional factors (e.g., the importance of the location of the symptoms) besides those previously identified in retrospective studies of patients with RA. Together with the data from previous research, these findings will help inform future health interventions aimed at increasing knowledge of RA and encouraging help-seeking. (First Release Feb 1 2015; J Rheumatol 2015;42:585-92; doi:10.3899/jrheum.140913)
\end{abstract}

Key Indexing Terms: PERCEPTIONS HELP-SEEKING

GENERAL PUBLIC

KNOWLEDGE RHEUMATOID ARTHRITIS

For optimal clinical outcomes, it is important that disease-modifying antirheumatic drug treatment is started within 3 months of the onset of rheumatoid arthritis (RA) symptoms ${ }^{1,2,3}$. Unfortunately, this vital window of opportunity is often missed because of delays at various stages of the patient's journey, in particular delays in initial help-seeking ${ }^{4,5,6,7,8}$.

In addition to the benefits of rapid treatment at an

From the Centre for Translational Inflammation Research, School of Immunity and Infection, College of Medical and Dental Sciences, The University of Birmingham; Sandwell and West Birmingham Hospitals National Health Service (NHS) Trust, Birmingham; Arthritis Research United Kingdom Primary Care Centre, Research Institute for Primary Care and Health Sciences, Keele University, Keele; and the Faculty of Medical and Human Sciences, School of Nursing, University of Manchester, Manchester, UK.

Supported by The Dunhill Medical Trust (grant number R226/1111). The authors further acknowledge the support of the National Institute for Health Research through the Primary Care Research Network. Dr. Mallen is funded by an Arthritis Research UK Clinician Scientist award.

G. Simons, Research Fellow, PhD; R.J. Stack, Research Fellow, PhD, Centre for Translational Inflammation Research, School of Immunity and Infection, College of Medical and Dental Sciences, The University of Birmingham; C.D. Mallen, Professor of General Practice Research, PhD, Arthritis Research UK Primary Care Centre, Research Institute for

Primary Care and Health Sciences, Keele University; K. Kumar, National individual level, there are also economic benefits at a societal level. In the United Kingdom, it has been estimated that doubling the proportion of patients with RA treated within 3 months of symptom onset from a currently estimated $10 \%$ would lead to significant cost savings (around $£ 2$ million per yr for the health service). In addition, there will be significant economic benefits from reduced sick leave and employment loss ${ }^{9}$.

Institute for Health Research Clinical Doctoral Research Fellow, MSc, Centre for Translational Inflammation Research, School of Immunity and Infection, College of Medical and Dental Sciences, The University of Birmingham, and the Faculty of Medical and Human Sciences, School of Nursing, University of Manchester; K. Raza, Professor of Clinical Rheumatology, Honorary Consultant Rheumatologist, PhD, Centre for Translational Inflammation Research, School of Immunity and Infection, College of Medical and Dental Sciences, The University of Birmingham, and the Sandwell and West Birmingham Hospitals NHS Trust.

Rebecca Jayne Stack and Karim Raza are joint senior authors of this report.

Address correspondence to Dr. G. Simons, Centre for Translational Inflammation Research, School of Immunity and Infection, College of Medical and Dental Sciences, The University of Birmingham, BirminghamB15 2TT,UK.E-mail: g.simons@bham.ac.uk.

Full Release Article. For details see Reprints/Permissions at jrheum.org. Accepted for publication December 10, 2014.

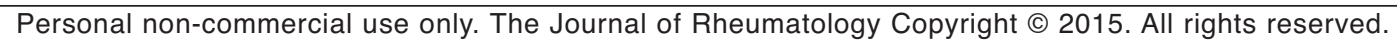


It is therefore important to develop effective health interventions that increase the general public's knowledge of RA and encourage those with new symptoms to seek medical attention. To do so, we need to understand the reasons for patient delay at RA onset. A metasynthesis of qualitative studies ${ }^{10}$ identified several factors related to patient delay, including the nature of symptom onset and the way that people interpret and deal with their symptoms. In addition, speaking to others and attitudes toward healthcare professionals were also reported to have an effect.

Since the publication of the metasynthesis, there have been a few further qualitative studies looking at the delay in help-seeking ${ }^{11,12}$. Patients with RA interviewed within a year of diagnosis described using over-the-counter medications prior to, or instead of, seeking medical advice or while awaiting a formal diagnosis ${ }^{11}$. In another set of interviews, factors prompting general practitioner (GP) consultation included a sudden and severe symptom onset, symptoms resistant to self-management strategies, symptoms not easing, symptoms disrupting daily activities, and the perception that symptoms were unusual and could not be explained ${ }^{12}$.

However, qualitative studies capturing the views of patients at the time of, or soon after, diagnosis are relatively rare $^{11,12,13,14}$. Most of the research discussed in the metasynthesis relied on responses from patients with longstanding disease ${ }^{15}$. A potential problem with this type of retrospective research (even within $1 \mathrm{yr}$ of symptom onset) is that a person's recall of past events can change over time ${ }^{16}$, and the responses given by patients with RA about their reasons for help-seeking at symptom onset might have been distorted by their experiences with the illness over time and postdiagnosis in particular ${ }^{17}$. To fully understand why some patients with RA do not seek help rapidly, we would ideally interview patients destined to develop RA within weeks of symptom onset. However, the entirety of this population is almost impossible to access since a large proportion of it would not have engaged with a healthcare professional within this time period.

When people experience the first symptoms of RA, their knowledge and perceptions will be very similar to those of many members of the general public. Thus, using hypothetical explorations to understand how individuals who have not seen a GP about RA symptoms would respond to the onset of RA symptoms avoids some of the issues described above. In addition, such research will provide insights into the knowledge and perceptions of RA in the general public prior to encountering RA symptoms as well as their perceived barriers to help-seeking. This, in addition to the findings from research with patients with RA, could help the development of interventions to increase the knowledge of RA and other musculoskeletal disorders, and decrease the delay in help-seeking at symptom onset, ultimately reducing healthcare costs and improving clinical outcomes.

At present, little is known about the perceptions and knowledge of RA among the general public ${ }^{4}$, and we do not know whether the same barriers to and drivers of help-seeking identified retrospectively by people with RA are identified by those who have not had these experiences. It is expected that although there will be an overlap between the findings from both types of study, certain findings from the studies with patients with RA will be colored by their subsequent experiences and thus not offer the whole picture. Therefore, our current qualitative study investigated how members of the general public without a diagnosis of RA perceive the illness and its symptoms, and what they believe might encourage or delay help-seeking if they were to develop symptoms of RA. This will allow us to make comparisons with the barriers and drivers identified in the research with patients with RA, substantiate and elaborate on those findings, and make recommendations for effective health interventions.

\section{MATERIALS AND METHODS}

Materials and methods. Ethical approval for the study was obtained from the South West-Bristol Research ethics committee (REC ref 12/SW/0195) and all participants provided written consent.

Participants. Patients registered at 2 inner city general practices, aged 18 years and over, and without a diagnosis of inflammatory arthritis (including RA) were invited to participate in a study "looking at perceptions of chronic illnesses such as arthritis in the general public". Participants were purposively sampled from 3 age groups (18-40, 41-60, and over 61 yrs) with the largest group being the 41-60 age group, allowing the final sample to demographically reflect the age distribution for RA onset. The study was additionally advertised through posters at the universities of Keele and Birmingham. Thirty-two individuals recruited through the general practices and 6 individuals recruited through the universities consented to an interview $(\mathrm{n}=38)$. The interview data from 1 additional participant could not be used because of recording problems. Participants (32 women) were aged between 20 years and 85 years (mean age 58, age of 1 participant unknown) and all but 1 were of a white ethnic background. Ten participants had a paramedical (e.g., nursing/physiotherapy) background and $21 \mathrm{knew}$ someone they thought had RA (Table 1).

Interview procedure and analysis. Semistructured interviews were conducted (by GS) in general practices, at the universities of Birmingham and Keele, or in participants' homes. Interviews lasted about $50 \mathrm{~min}$ and were guided by an interview schedule developed in collaboration with patient research partners and informed by previous research ${ }^{10,18,19}$ Questions explored participants' anticipated reactions to descriptions of early RA symptoms. For example, participants were asked what they "would think if [they] woke up one morning and [their] joints were stiff" and subsequently what they "would do if this stiff feeling did not disappear for over an hour" (similar questions were asked for joint pain and swelling. Table 2).

The interviews were audio-recorded and transcribed verbatim. Initial blind coding of 3 randomly selected transcripts was undertaken by RJS and GS. As a result of a good intercoder agreement, no further blind coding of additional manuscripts was deemed necessary and any discrepancies in coding were discussed and resolved prior to the coding of the other transcripts by GS. Additional codes emerging from this subsequent coding were checked by RJS and discussed. The coded interview data were analyzed using thematic analysis ${ }^{20}$ supported by NVivo10 software $^{21}$ and involved searching for meaningful themes amid the coded data. Emerging themes were discussed with KK and KR. The validity of the final selection of themes and supporting quotations was checked by RJS.

\section{RESULTS}

Barriers to and drivers of help-seeking. The themes

Personal non-commercial use only. The Journal of Rheumatology Copyright (C) 2015. All rights reserved. 
Table 1. Participant demographic characteristics.

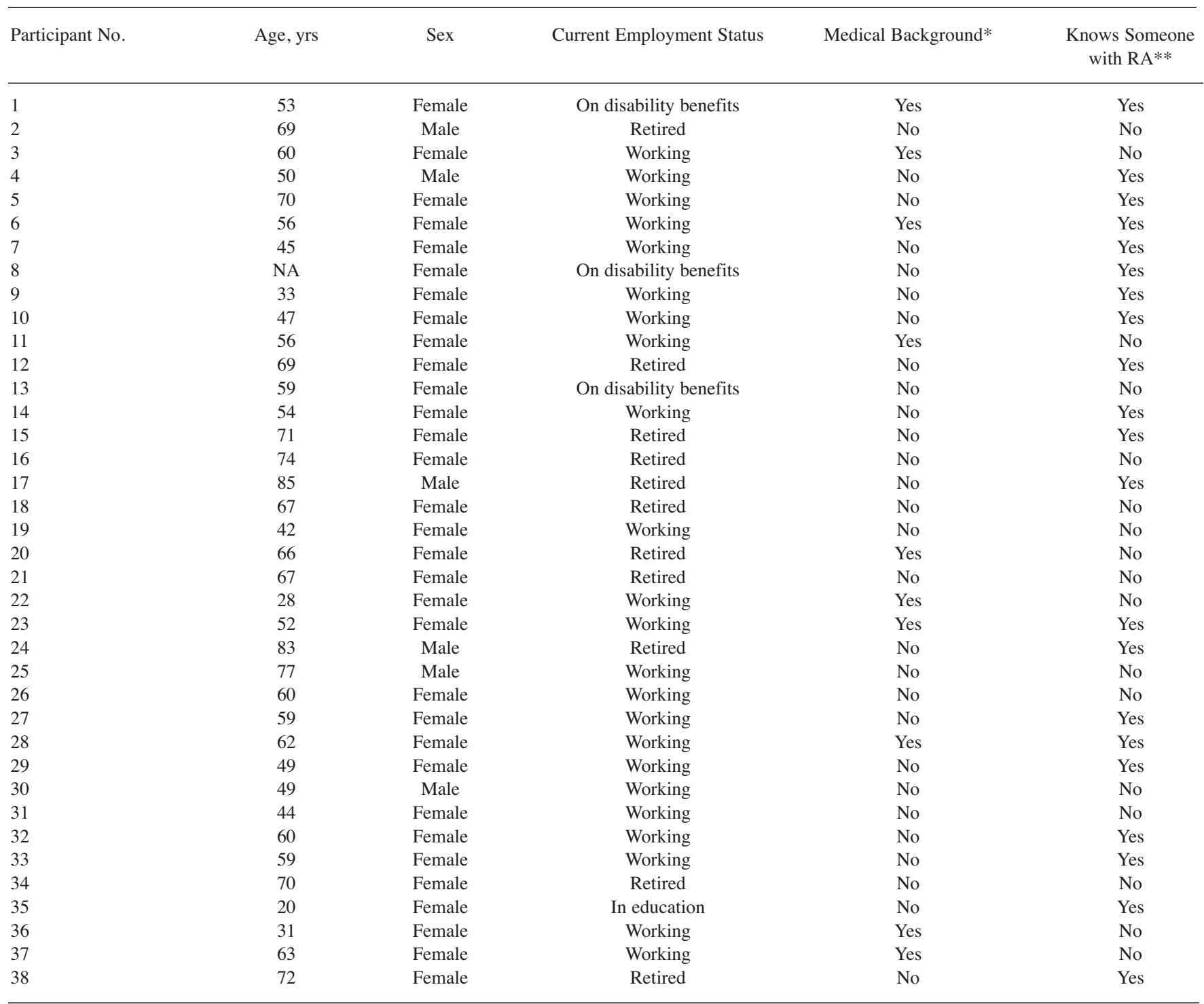

* These participants either currently work in healthcare (e.g., as a physiotherapist or nurse) or otherwise have a medical background. ** As indicated by the participant. RA: rheumatoid arthritis.

Table 2. Sample questions from the interview schedule.

Cues to action. Swollen joints (similar questions for joint pain and joint stiffness).

- What would you think if you noticed your fingers were swollen? What would you attribute swollen fingers to?

- What would you do about it?

- Would swollen fingers worry you?

- At which point would you go to your GP or seek help elsewhere?

- If your feet were swollen, would you feel differently or would your actions be different?

Barriers to help-seeking.

- In general, do you feel able to go to your GP?

- What would stop you from going to the GP with these symptoms?

- What would encourage you to go to your GP with these symptoms?

Note: these are example questions only. The interview schedule was used only as a guideline. The wording of the questions varied between interviews. GP: general practitioner. 


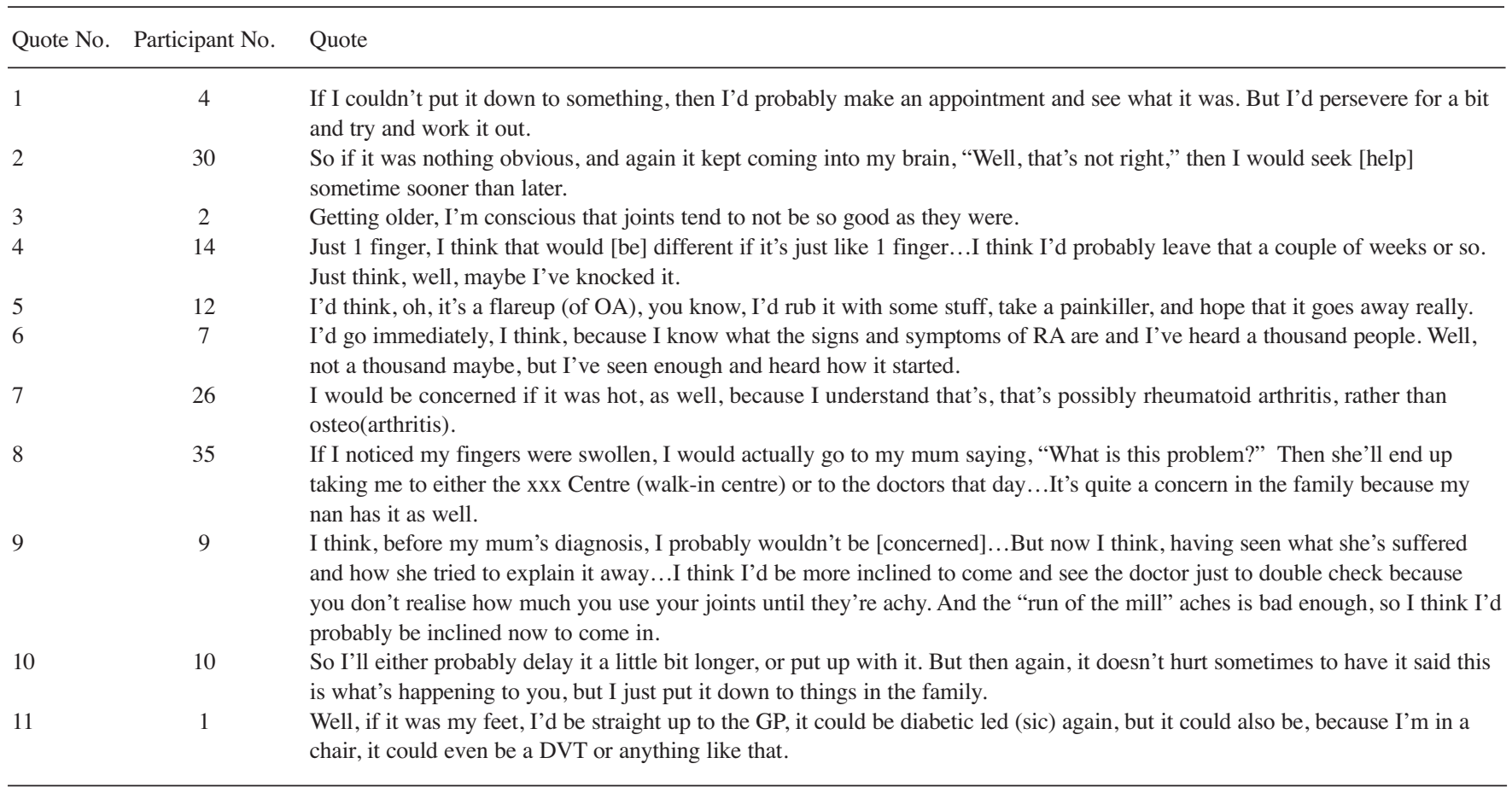

RA: rheumatoid arthritis; OA: osteoarthritis; GP: general practitioner; DVT: deep vein thrombosis.

Table 4. Quotes related to theme 2: the presentation, location, and experience of joint symptoms.

\begin{tabular}{lcl}
\hline Quote No. Participant No. & Quote \\
\hline 1 & 31 & $\begin{array}{l}\text { I'd try and leave it as long as possible, because I'd always have the hope that it will get better or sort itself out. } \\
\text { But, again, it (whether I do something) depends if it's a reocurring pattern and for how long. } \\
\text { But if it was unusual for me, although it wasn't significantly impacting my life, I'd probably wait around about a week before } \\
\text { going to get.... medical opinion. }\end{array}$ \\
4 & 36 & $\begin{array}{l}\text { Depends on how severe it was. I mean, if I was just aching...I just tend to put it to the back of my mind and carry on what } \\
\text { I'm doing. }\end{array}$ \\
5 & 3 & $\begin{array}{l}\text { If it's } 2 \text { knees, or it's more than 1 joint. I'm thinking, “Oh, it's something more likely to continue and not get better". } \\
\text { Your hands you can cope with...But if you can't tolerate walking, or tolerate standing, then you are really decreasing your } \\
\text { standard of living. I wouldn't mess about if my feet started aching. }\end{array}$ \\
7 & 23 & $\begin{array}{l}\text { If I was in pain, I'm quicker to come in [to the GP]. If it's not painful, then I probably wouldn't and I haven't. } \\
\text { I think mine's quite high, my pain level. I think the more pain you get and the more severe it is, you do tend to cope with it a } \\
\text { bit better. }\end{array}$ \\
\hline
\end{tabular}

GP: general practitioner.

presented here reflect the reasons for rapid or delayed help-seeking discussed in the interviews. Five themes were identified: (1) perceived causes of symptoms; (2) factors related to the presentation, location, and experience of symptoms; (3) perceived effect of symptoms on daily life; (4) self-management of symptoms; and (5) GP-related drivers and barriers. Illustrative quotes can be found in Tables 3, 4, 5, and 6 .

1. Perceived causes of symptoms. When considering their response to the description of symptoms, such as joint pain, many participants described trying to identify external causative factors and suggested that they would put up with symptoms while attempting to establish their potential cause (Table 3, Quote 1, further referred to as T3Q1). For others, the lack of an obvious cause for their symptoms would drive rapid help-seeking (T3Q2).

Several participants felt that joint pain and stiffness were simply part of getting older and as a result, they would delay or avoid seeking medical attention (T3Q3). Others mentioned "knocks" and other injuries as a possible cause, specifically when experiencing symptoms in a single joint. They would delay help-seeking if they suspected injury as a causal factor (T3Q4).

Many participants identified the symptoms presented to 
Table 5. Quotes related to themes 3 and 4 .

\begin{tabular}{|c|c|c|}
\hline & cipant No. & Quote \\
\hline \multicolumn{3}{|c|}{ Theme 3: Perceived effect on daily life. } \\
\hline 1 & 36 & $\begin{array}{l}\text { If it began to interfere with my day-to-day life, I would go sooner than that (a week) because I probably couldn't sustain it } \\
\text { for that period of time if it was interfering with coming to work or, you know, doing the usual household tasks. }\end{array}$ \\
\hline 2 & 11 & $\begin{array}{l}\text { Now that might get me somewhere because you'd be struggling round the kitchen, trying to get your breakfast and dressing } \\
\text { yourself, and that would actually affect your lifestyle... }\end{array}$ \\
\hline 3 & 6 & $\begin{array}{l}\text { Well, I think if it was keeping me awake at night and I wasn't getting my sleep and it was stopping me [from] doing things, } \\
\text { then I probably would go quite quickly. If it was just not causing me much trouble, then I probably would leave it. }\end{array}$ \\
\hline 4 & 17 & $\begin{array}{l}\text { I would use the painkillers for a while and then if...it's getting worse, I've got enough common sense to know if things } \\
\text { aren't right, not for just a short time, but over a period of time if things don't improve. It's like, if you get a cold or it turns } \\
\text { to [the] flu, eventually you've got to go and see the doctor. }\end{array}$ \\
\hline 5 & 1 & $\begin{array}{l}\text { If it (morning stiffness) was around after an hour, I'd probably, again, I'd be more inclined to actually go and have a shower. } \\
\text { Because I'm not a fan of running for tablets. I take enough as it is, so I'm also a firm believer in that nature offers its own } \\
\text { remedies and I do find having a good hot shower will loosen me up. }\end{array}$ \\
\hline
\end{tabular}

Table 6. Quotes for theme 5: GP-related drivers and barriers.

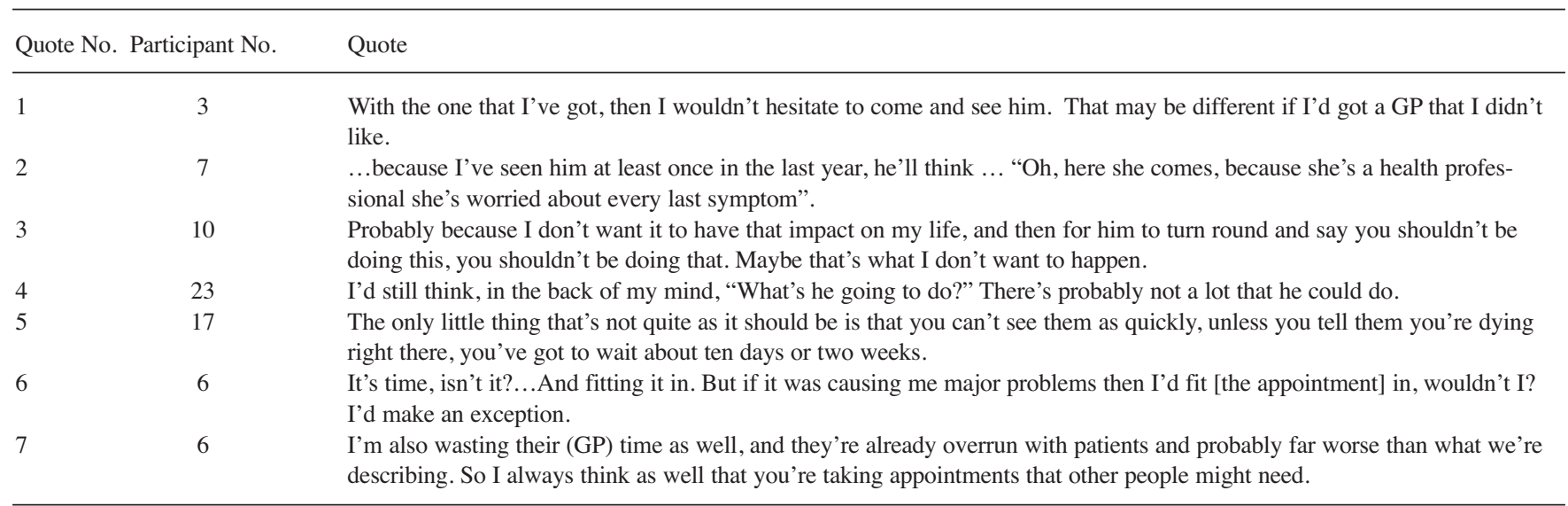

GP: general practitioner.

them simply as symptoms of "arthritis". Frequently, the symptoms were attributed to what is likely to be osteoarthritis (OA), or to "wear and tear", rather than RA. Participants often related the symptoms to their own experiences of musculoskeletal symptoms and would see the symptoms as an extension of these symptoms rather than a different illness, an attitude that in turn might delay help-seeking (T3Q5).

In contrast, those participants who did identify the symptoms as indicative of RA usually perceived RA as a serious illness and indicated that they would seek help rapidly (T3Q6). Some indicated that they were aware of the differences between OA and RA (T3Q7). For several participants, recognizing the symptoms as being indicative of RA resulted from them having seen a family member or friend with RA. Some would seek help rapidly for similar symptoms as a result (T3Q8), especially if they witnessed the consequences of delays in help-seeking (T3Q9). Others did not appear to take this personal experience into consid- eration when responding to the description of RA symptoms and indeed admitted poor knowledge about RA despite knowing someone with the illness. For a few, having a relative with RA would in fact delay help-seeking (e.g., if they perceived it to be inevitable that they would develop RA because of their family history; T3Q10).

Finally, participants' own existing nonarticular health problems (e.g., diabetes) also affected the participants' interpretations of the causes of the symptoms presented to them. Several participants indicated that symptoms such as swelling or stiffness could be part of their existing non-musculoskeletal illness and this might be a reason for rapid help-seeking (T3Q11).

2. Factors related to the presentation, location, and experience of symptoms. Many participants indicated that if symptom onset was gradual (as opposed to sudden), they would wait for anything between a few days and several weeks, but that they would seek medical attention if the symptoms did not abate. Some participants would put up

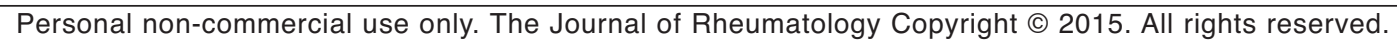


with symptoms for a long time hoping that they would resolve spontaneously (T4Q1).

For others, palindromic symptoms (T4Q2) or perceiving the symptoms as new or unusual (T4Q3) would cause them to seek help quickly. For many, symptom severity would play a major role in their help-seeking decision (T4Q4). Participants further mentioned that they would be more likely to seek medical attention if they were experiencing symptoms in multiple joints rather than a single joint (T4Q5).

The location of the symptoms was also important. Whereas some would seek help sooner if the symptoms occurred in their feet, because of the effect on mobility (T4Q6), others perceived symptoms in the hands to be more worrisome.

Finally, although each of the classic symptoms of RA (joint pain, swelling, and stiffness) was discussed during the interviews, joint pain was an especially important trigger for seeking medical attention (T4Q7), although some cited their "tolerance of pain" as a reason for not seeking help (T4Q8).

3. Perceived effect on daily life. Many participants said they would take action if and when symptoms had an effect on their daily activities, especially in relation to work (T5Q1 and T5Q2). For example, 1 participant saw multiple functional implications of stiff joints and indicated that it would cause her to seek help (T5Q3).

4. Self-management of symptoms. Many participants would try to self-manage the symptoms at first, for example by making lifestyle adjustments, massaging the affected joint, using topical and oral over-the-counter treatments such as analgesics (T5Q4), or using other (alternative) treatments (T5Q5). Participants who would initially choose to self-manage their symptoms wanted to give these approaches some time to work before seeking help (T5Q6).

5. GP-related drivers and barriers. Many participants indicated that on the whole they felt comfortable going to see their GP (T6Q1), although some participants did worry about how they were viewed by their GP (T6Q2).

For some, reluctance to see their GP was the result of perceived negative consequences, such as the GP telling them to refrain from certain activities or hobbies (T6Q3), or prescribing undesired medication. Participants further varied in their perceptions of the availability of effective treatments, and this could also affect help-seeking (T6Q4).

Participants also described potential delays caused by difficulties in obtaining appointments (T6Q5) or fitting a GP appointment into their daily routine (T6Q6). Some participants would delay help-seeking because they perceived the symptoms to be fairly trivial and would not want to take up an appointment "unnecessarily" or waste the GP's time (T6Q7).

\section{DISCUSSION}

This exploration of the views held by members of the general public without RA revealed a number of potential barriers to and drivers of help-seeking for symptoms of RA. These can be summarized by 5 overarching themes: perceived cause of symptoms; presentation, location, and experience of symptoms; perceived effect of symptoms on daily life; planned self-management of symptoms; and GP-related drivers and barriers.

Correct assessment of the cause of symptoms has been shown to shorten delay in other diseases, for example, cancers $^{22}$. However, similar to patients with RA in previous research $^{10,23,24}$, many of the participants in our present study would misattribute the cause of classic RA symptoms to aging, "wear and tear", or OA, and as a result would delay help-seeking. Those participants who associated the symptoms presented with RA and understood that effective treatment was available would generally seek help as a matter of urgency. Knowing someone with RA could aid correct causal assessment and subsequent rapid help-seeking. Indeed, in contrast to what was found in previous research with patients with $\mathrm{RA}^{23}$, at least some of our participants indicated that this personal connection would lead them to take swift action. However, others with similar connections would not do so, because they perceived it as unavoidable that they would get RA, for example. Thus, although knowing someone with RA might enhance the knowledge of RA, its symptoms, and consequences, this personal experience might not necessarily lead to rapid help-seeking. These contrasts echo those found in research with patients with cancer ${ }^{22}$. Knowing someone with cancer might cause some people to delay help-seeking, for example because they think that the cancer is hereditary and therefore unavoidable, or when the personal experience had been traumatic ${ }^{25}$. The current research further shows that a person's own experiences with musculoskeletal symptoms, as well as nonarticular health problems (e.g., diabetes), would influence their interpretation of the cause of the symptoms and subsequent help-seeking. If the first symptoms of RA are misinterpreted as being the result of an existing illness, a patient might first try to manage the symptoms of RA in the same way as they manage their existing condition. Alternatively, they might see any changes to their condition as urgent and seek help accordingly.

When looking at factors related to the presentation, location, and experience of symptoms, the mode of symptom onset, severity, and progression of symptoms were clear triggers, as well as was the location of the symptoms. The latter factor has not been identified in the metasynthesis of research with patients with $\mathrm{RA}^{10}$, but seems to drive the perceptions of those without RA and their envisaged course of action. Similar to many patients with $\mathrm{RA}^{12,23}$, participants would wait until symptoms affected daily activities before seeking medical attention, which might lead to long delays in help-seeking (especially with a slow onset of symptoms). Further, echoing the intentions of some patients

Personal non-commercial use only. The Journal of Rheumatology Copyright (C) 2015. All rights reserved 
with $\mathrm{RA}^{11}$, several participants proposed to self-manage the symptoms, which might again cause delays in help-seeking because people would wait to see whether their approaches had the desired effect ${ }^{12}$.

Finally, similar to a large proportion of patients with $\mathrm{RA}^{23}$, most participants had a good relationship with their GP and felt that they were able to consult with their GP in connection with the presented symptoms. However, others mentioned that the way they would be viewed by their GP, the perception that they would waste the GP's time with something trivial, or their fear of what the GP might say or do would have a negative effect on their help-seeking behavior.

In general, the findings from our current research substantiate those from studies of patients with $\mathrm{RA}^{10,11}$, particularly findings related to self-managing symptoms, attributing symptoms to aging, and responding to the nature of symptom onset. The similarities between our findings and the findings of previous research studies involving patients with RA highlight that the use of hypothetical scenarios in members of the public without the disease represents an approach that can elicit comparable and valid findings. Further, some additional factors (not identified in previous research), such as the location of symptoms, were high-lighted by our study as potentially playing a role in the decision to seek help. These findings help to explain why long patient delays in help-seeking are often observed ${ }^{5,8}$, and should inform the development of any public health intervention aimed at improving knowledge of RA and minimizing barriers to help-seeking in the following ways. First, to address the misattributions related to the cause of the symptoms and the resulting delays, it is important that the general public is informed about the symptoms of inflammatory arthritis and the differences between RA, OA, and other causes of musculoskeletal symptoms. Such messages should further highlight the significance of all RA symptoms, the fact that these symptoms might not all occur simultaneously or in specific joint(s), and that even mild symptoms could herald the onset of a severe disease ${ }^{26}$. Highlighting the potential seriousness of RA, even when the symptoms begin mildly or gradually ${ }^{10}$, might also stop people from feeling that they are "time wasters," thus removing another barrier to a GP visit ${ }^{27}$.

Second, although self-management of symptoms might be beneficial for the patient in the short term (e.g., pain management until a GP appointment), it should be stressed that this should not delay help-seeking. There might be an important role for the pharmacist. When asked for oral or topical analgesics or nonsteroidal antiinflammatory medications, pharmacists should be ready to question the patients about their symptoms and direct them to make appointments with their GP where appropriate. Third, the public needs to be better informed about the fact that treatments for RA are available and that early treatment of RA symptoms reduces the risk of longterm joint damage and disability ${ }^{1}$.
An important challenge to the development of an effective public health campaign for RA relates to the multiple different symptoms that herald the onset of RA and that can occur in varied combinations. Qualitative research involving patients with seropositive arthralgia at risk of RA or with new onset RA found that besides joint pain, swelling, warmth, and stiffness, patients experienced a range of other symptoms including weakness, loss of motor control, fatigue, sleeping difficulties, and depressive symptoms early in their disease ${ }^{28,29}$. Identifying a simple constellation of symptoms that recognizes the majority of early RA presentations (i.e., that is appropriately sensitive) and yet does not detect an inappropriately large proportion of the general population (i.e., is appropriately specific) will be challenging, but is important for maximizing appropriate help-seeking and minimizing inappropriate help-seeking ${ }^{10,27}$.

Our study has a number of limitations. First, the sample consisted mainly of participants from a white British background. Given previous research showing that patients with RA from the South Asian community in the United Kingdom had a poor understanding of RA and its treatments ${ }^{30}$, the current research should be extended to those from other ethnicities without a diagnosis of RA. Second, although we aimed for the sample to reflect the age and sex distribution (1 man for every 3 women) of the RA population, men were relatively underrepresented (1 man for every 5 women). Even though men are less likely to develop $\mathrm{RA}$, it is important to understand their perceptions about illness and help-seeking ${ }^{31}$ because there may be sex-specific issues. Third, a proportion of participants had a paramedical background and/or knew people with RA, which might have affected their perceptions and level of knowledge. However, many individuals with such connections still suggested that they would delay help-seeking or professed to having little to no knowledge of RA. Fourth, participants were presented with short descriptions of the main symptoms of RA (joint pain, stiffness, and swelling). It might have been difficult for them to imagine, for example, the type or intensity of joint pain experienced by some patients with RA. In addition, future research should look at other features of RA, such as fatigue. Finally, whether behavioral intentions expressed in response to hypothetical scenarios are translated into actual behaviors will depend in part on factors such as past behavior and the control the person feels they have over the behavior at the time that action is actually required ${ }^{32}$. These factors are difficult to measure in qualitative research and further survey and observational research is needed.

The results of our study, together with the results from the research with patients with $\mathrm{RA}^{10,11,12}$, help clarify the reasons underlying lengthy patient delay often observed in patients with a new onset of RA symptoms. There are several steps that can be taken to remove some of the barriers to GP consultation. Besides direct approaches, such as working with pharmacists to reduce the number of people

Personal non-commercial use only. The Journal of Rheumatology Copyright (c) 2015. All rights reserved. 
trying to self-manage their symptoms, the results and recommendations should be used to inform the content of health interventions and information materials aimed at increasing public awareness of RA, and the need for early consultation.

\section{ACKNOWLEDGMENT}

We thank the interviewees who took part in our study and the patient research partners who have been involved in the development of the research. In particular, we thank Dr. Marie Falahee, a member of the Birmingham Rheumatology Research Patient Partnership, for her contributions to the development of the interview schedule and suggestions for improvement of the current manuscript.

\section{REFERENCES}

1. Raza K, Buckley CE, Salmon M, Buckley CD. Treating very early rheumatoid arthritis. Best Pract Res Clin Rheumatol 2006; 20:849-63.

2. Finckh A, Liang MH, van Herckenrode CM, de Pablo P. Long-term impact of early treatment on radiographic progression in rheumatoid arthritis: a meta-analysis. Arthritis Rheum 2006; 55:864-72.

3. van der Linden MP, le Cessie S, Raza K, van der Woude D, Knevel $\mathrm{R}$, Huizinga TW, et al. Long-term impact of delay in assessment of patients with early arthritis. Arthritis Rheum 2010;62:3537-46.

4. Stack RJ, Simons G, Kumar K, Mallen CD, Raza K. Patient delays in seeking help at the onset of rheumatoid arthritis: the problem, its causes and potential solutions. Aging Health 2013;9:425-35.

5. Kumar K, Daley E, Carruthers DM, Situnayake D, Gordon C, Grindulis K, et al. Delay in presentation to primary care physicians is the main reason why patients with rheumatoid arthritis are seen late by rheumatologists. Rheumatology 2007;46:1438-40.

6. Villeneuve E, Nam JL, Bell MJ, Deighton CM, Felson DT, Hazes JM, et al. A systematic literature review of strategies promoting early referral and reducing delays in the diagnosis and management of inflammatory arthritis. Ann Rheum Dis 2013;72:13-22.

7. Feldman DE, Bernatsky S, Houde M, Beauchamp ME, Abrahamowicz M. Early consultation with a rheumatologist for RA: does it reduce subsequent use of orthopaedic surgery? Rheumatology 2013;52:452-9.

8. van Nies JA, Brouwer E, de Rooy DP, van Gaalen FA, Huizinga TW, Posthumus MD, et al. Reasons for medical help-seeking behaviour of patients with recent-onset arthralgia. Ann Rheum Dis 2013;72:1302-7.

9. National Audit Office. Services for people with rheumatoid arthritis. Report by the comptroller and auditor general, HC 823 Session 2008-2009. [Internet. Accessed December 30, 2014.] Available from: www.nao.org.uk/wp-content/uploads/2009/07/ 0809823.pdf

10. Stack RJ, Shaw K, Mallen C, Herron-Marx S, Horne R, Raza K. Delays in help seeking at the onset of the symptoms of rheumatoid arthritis: a systematic synthesis of qualitative literature. Ann Rheum Dis 2012;71:493-7.

11. Townsend A, Backman CL, Adam P, Li LC. A qualitative interview study: patient accounts of medication use in early rheumatoid arthritis from symptom onset to early postdiagnosis. BMJ Open 2013;3:e02164.

12. Townsend A, Backman CL, Adam P, Li LC. Women's accounts of help-seeking in early rheumatoid arthritis from symptom onset to diagnosis. Chronic Illn 2014;10:259-72.

13. Hay MC, Cadigan RJ, Khanna D, Strathmann C, Lieber E, Altman $R$, et al. Prepared patients: internet information seeking by new rheumatology patients. Arthritis Rheum 2008;59:575-82.
14. Bury M. Chronic illness as biographical disruption. Sociol Health Illn 1982;4:167-82.

15. Schneider M, Manabile E, Tikly M. Social aspects of living with rheumatoid arthritis: a qualitative descriptive study in Soweto, South Africa - a low resource context. Health Qual Life Outcomes 2008:6:54

16. Simons G, Parkinson B. Time-dependent observational and diary methodologies and their use in studies of social referencing and interpersonal emotion regulation. 21st Century Soc 2009;4:175-86.

17. Oliver S, Bosworth A, Airoldi M, Bunyan H, Callum A, Dixon J, et al. Exploring the healthcare journey of patients with rheumatoid arthritis: a mapping project - implications for practice. Musculoskeletal Care 2008;6:247-66.

18. Bishop GD, Converse SA. Illness representations: a prototype approach. Health Psychol 1986;5:95-114.

19. Cameron L, Leventhal EA, Leventhal H. Symptom representations and affect as determinants of care seeking in a communitydwelling, adult sample population. Health Psychol 1993;12:171-9.

20. Braun V, Clarke V. Using thematic analysis in psychology. Qual Res Psychol 2006;3:77-101.

21. NVivo qualitative data analysis software. QSR International Pty Ltd., Version 10, 2012.

22. Smith LK, Pope C, Botha JL. Patients' help-seeking experiences and delay in cancer presentation: a qualitative synthesis. Lancet 2005;366:825-31.

23. Townsend A, Adam P, Cox SM, Li LC. Everyday ethics and help-seeking in early rheumatoid arthritis. Chronic Illn 2010; 6:171-82.

24. Sheppard J, Kumar K, Buckley CD, Shaw KL, Raza K. 'I just thought it was normal aches and pains': A qualitative study of decision-making processes in patients with early rheumatoid arthritis. Rheumatology 2008;47:1577-82.

25. de Nooijer J, Lechner L, de Vries H. A qualitative study on detecting cancer symptoms and seeking medical help; an application of Andersen's model of total patient delay. Patient Educ Couns 2001;42:145-57.

26. Raza K, Saber TP, Kvien TK, Tak PP, Gerlag DM. Timing the therapeutic window of opportunity in early rheumatoid arthritis: proposal for definitions of disease duration in clinical trials. Ann Rheum Dis 2012;71:1921-3

27. Stack RJ, Llewellyn Z, Deighton C, Kiely P, Mallen CD, Raza K. General practitioners' perspectives on campaigns to promote rapid help-seeking behaviour at the onset of rheumatoid arthritis. Scand J Prim Health Care 2014;32:37-43.

28. Stack RJ, van Tuyl LH, Sloots M, van de Stadt LA, Hoogland W, Maat B, et al. Symptom complexes in patients with seropositive arthralgia and in patients newly diagnosed with rheumatoid arthritis: a qualitative exploration of symptom development. Rheumatology 2014;53:1646-53.

29. Stack RJ, Sahni M, Mallen CD, Raza K. Symptom complexes at the earliest phases of rheumatoid arthritis: A synthesis of the qualitative literature. Arthritis Care Res 2013;65:1916-26.

30. Kumar K, Daley E, Khattak F, Buckley CD, Raza K. The influence of ethnicity on the extent of, and reasons underlying, delay in general practitioner consultation in patients with RA. Rheumatology 2010;49:1005-12.

31. Galdas PM, Cheater F, Marshall P. Men and health help-seeking behaviour: literature review. J Adv Nurs 2005;49:616-23.

32. Ajzen I. The theory of planned behavior. Organ Behav Hum Decis Process 1991;50:179-211. 